\title{
Femto-Molar Sensitive Field Effect Transistor Biosensors Based on Silicon Nanowires and Antibodies
}

\author{
F. Puppo*, M.-A. Doucey ${ }^{\dagger}$, T.S.Y. Moh ${ }^{\ddagger}$, G. Pandraud ${ }^{\ddagger}$, P.M. Sarro ${ }^{\ddagger}$, G. De Micheli* ${ }^{*}$, S. Carrara* \\ *Integrated System Laboratory, EPFL-École Polytechnique Fédérale de Lausanne \\ 1015 Lausanne, Switzerland \\ Email: francesca.puppo@epfl.ch \\ ${ }^{\dagger}$ Ludwig Center for Cancer Research, UNIL-Université de Lausanne \\ 1066 Epalinges, Switzerland \\ Email: Marie-Agnes.Doucey@unil.ch \\ $\ddagger$ Delft Institute of Microsystems and Nanoelectronics (DIMES), TU Delft-Delft University of Technology \\ 2628 CT Delft, The Netherlands \\ Email: t.s.y.moh@tudelft.nl
}

\begin{abstract}
This article presents electrically-based sensors made of high quality silicon nanowire field effect transistors (SiNWFETs) for high sensitive detection of vascular endothelial growth factor (VEGF) molecules. SiNW-FET devices, fabricated through an IC/CMOS compatible top-down approach, are covalently functionalized with VEGF monoclonal antibodies in order to sense VEGF. Increasing concentrations of VEGF in the femto molar range determine increasing conductance values as proof of occurring immuno-reactions at the nanowire (NW) surface. These results confirm data in literature about the possibility of sensing pathogenic factors with SiNW-FET sensors, introducing the innovating aspect of detecting biomolecules in dry conditions.
\end{abstract}

\section{INTRODUCTION}

Early detection of important diseases such as cancer is essential to determine appropriate treatments in order to effectively cure the malady. However, current diagnostic tools show critical constraint regarding the detection of biomarkers in amounts still far away from the very low concentrations characterizing the early stage of the diseases.

Devices like field-effect transistors (FETs) can be suitable candidates for sensors and diagnosis tools. Thanks to their ability to directly translate the interaction with target molecules on their surface into a readable signal, and to their compatibility with advanced micro- and nano-fabrication technology, FET devices are attractive to realize label-free, fast, simple, inexpensive, and real-time analysis of bioanalytes [1].

In particular, SiNW-FETs have been demonstrated very promising tools for biosensing purposes thanks to the unique tunable electrical and chemical properties of NWs. Indeed, the electrical properties and sensitivity of nanowires can be tuned reproducibly by controlling dopant type and concentration, and NW diameter [2]. In addition, given the existing massive knowledge on the chemical modification of silicon oxide surfaces [3], [4], high-performance SiNW-FET biosensors can be realized by linking recognition groups to the surface of the nanowire [5], [6], [7]. Finally, SiNWs can also benefit from existing and mature silicon industry for mass production and thus, be easily integrated with the well-developed field-effect transistor (FET) technology for low-cost, high-sensitive, pointof-care diagnosis systems.

In this work, a sensor based on SiNW-FET and surface functionalization with antibodies has been developed and used to sense vascular endothelial growth factor (VEGF). VEGF is a promoter for the formation of blood vessels in embryogenesis and wound healing, and it is also a major regulator of pathological angiogenesis. Angiogenesis is highly active in tumor growth, and various ischemic and inflammatory diseases. These biological properties make VEGF an important therapeutic target and biomarker of serious diseases [8], [9]. The early detection of biomarkers such as VEGF levels in patient blood, and the identification of their role in human diseases represent a very important challenge.

In the present article we demonstrate that CMOS compatible SiNW based sensors can detect pathological factors like VEGF in the femto molar range. Our approach takes advantage of the dry conditions under which electrical measurements are performed, which let us getting rid of the Debye screening problem strongly affecting the performances in standard FET sensors in liquid.

\section{PRINCIPLE OF OPERATION}

Fig. 1 illustrates a schematic model of the SiNW-FET based immunosensor. The FET nanowire channel is modified with antibodies acting as molecular probes. The biosensor is then incubated in a solution of antigen molecules. When complementary antigen species are found in close proximity of the wire surface, antigens bind to the antibodies previously deposited. In standard FET based biosensors, the detection signal resulting from the formation of antibody-antigen couples is measured in liquid buffer solutions. One major problem 


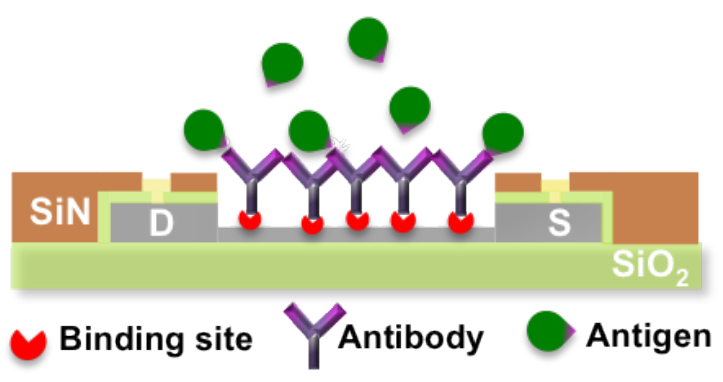

Fig. 1. Model of the NW-FET based biosensor.

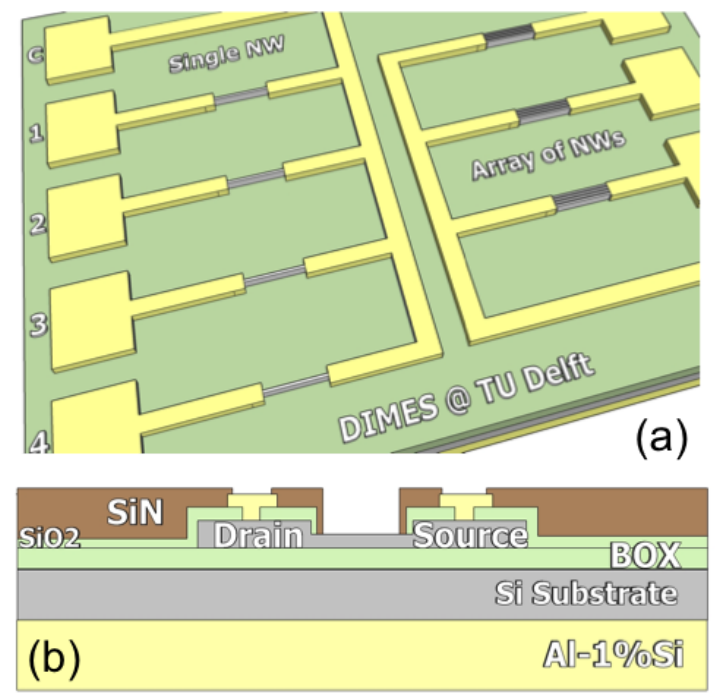

Fig. 2. (a) Schematic drawing of SiNW-FETs on a $10 \mathrm{~mm} \times 15 \mathrm{~mm}$ die size. (b) Cross section of a SiNW-FET device with a back gate configuration.

of sensing in electrolytic solutions is leakage currents and undesirable electrochemistry at the FET-liquid interface [10]. The interaction potential between the receptors and analytes that causes the conductance change in the FET sensor is partially screened by the strong ionic strength of the buffer solution. This screening directly depend on the Debye-Hückel length [11], [12].

In the present approach, we get rid of the Debye screening problem by performing electrical measurements on samples that have been dried after occured uptake [13]. The environment conditions, such as humidity and temperature, affect the sensing [14]; thus, all measurements have been performed under controlled chamber conditions.

Varying conductivity properties of the SiNW-FET sensor demonstrates charge redistribution and surface potential variations in the wire [15] as a consequence of occurring immunorecognition events. Improved sensitivity in dry conditions is ensured by an increased Debye length deriving from the absence of counterions from the electrolytic solution.

\section{SENSOR FABRICATION AND TEST}

\section{A. Fabrication of SiNW-FETS}

The SiNW-FET sensor was fabricated using conventional IC/CMOS compatible technology in Class 100 at Delft In- stitute of Microsystems and Nanoelectronics (DIMES), Delft University of Technology.

A conventional 4-inch SOI wafer from SOITEC France was used. The starting material of the SOI wafer has a $340 \mathrm{~nm}$ top silicon layer with a buried oxide layer of $400 \mathrm{~nm}$ of thickness. To create a P-type FET devices, the body of the SiNW was implanted with Boron and has doping concentration in the range of $10^{-16}-10^{-17} \mathrm{~cm}^{-3}$. The contact region was further implanted with higher concentration $\left(10^{-19} \mathrm{~cm}^{-3}\right)$ of similar dopants for low Ohmic contact which will subsequently form Source and Drain regions. To form a three terminal device and achieve the requirement of having the sensor open for biosensing, the gate terminal is formed at the backside of the device as illustrated in Fig. 2. The definition/patterning of the NW was done using standard photolithography resulting in micron size slab and gradually being reduced down to $100 \mathrm{~nm}$ wide using low etch rate AZ400k developer compromising of $15 \%$ of Potassium Borate in water. The etching was done in room temperature and no high temperature processing step is involved. The details of the process was first published by Moh et al. [16], [17], [18].

\section{B. Bio-functionalization}

The fabricated SiNW-FETs were converted to biosensors by surface modification with antibodies at the Ludwig Center for Cancer Research of the University of Lausanne, UNIL.

Chemicals unless stated otherwise were purchased from Sigma-Aldrich (St-Louis, MO). The silicon nanowires were functionalized by covalent attachment of anti-VEGF monoclonal antibody (R\&D Systems, clone 26503) with GPTS (glycidoxypropyltrimethoxysilane) [19] using a modification of the procedure described by Kim et al. [20]. The SiNW surface was cleaned with piranha 1:1 solution $\left(\mathrm{H}_{2} \mathrm{SO}_{4} / \mathrm{H}_{2} \mathrm{O}_{2}\right)$, dried and incubated for $60 \mathrm{~min}$ at RT (Room Temperature) in ethanol containing $10 \mathrm{mM}$ acetic acid and 1\% GPTS. Following extensive washes with ethanol/ acetic acid solution, the surface was dried under a $\mathrm{N}_{2}$ stream and placed for 15 min at $11^{\circ} \mathrm{C}$ in a dried oven. The surface was cooled down at RT and incubated over night in a humid chamber at RT with PBS (Phosphate Buffer Saline) containing $0.5 \mathrm{mg} / \mathrm{ml}$ of anti-VEGF solution. Following extensive washes with PBS, the remaining active GPTS-derived groups were blocked by ethanolamine $(10 \mathrm{mM}$ ethanolamine, $\mathrm{pH} 8.0)$ for $60 \mathrm{~min}$ at RT. The excess of ethanolamine was removed by PBS washes and the surface blocked by an additional incubation with PBS containing 3\% gelatin from cold water fish skin for $30 \mathrm{~min}$ at RT. The modified surface was washed and stored in PBS at $4^{\circ} \mathrm{C}$ until use.

\section{Sensor characterization}

After the fabrication, SiNW-FET based biosensors were characterized by electrical measurements in the Integrated System Laboratory at EPFL, Lausanne.

The nanowires were successively exposed for one hour at room temperature to solutions containing PBS and known concentrations of VEGF. Fluorescence imaging tests 

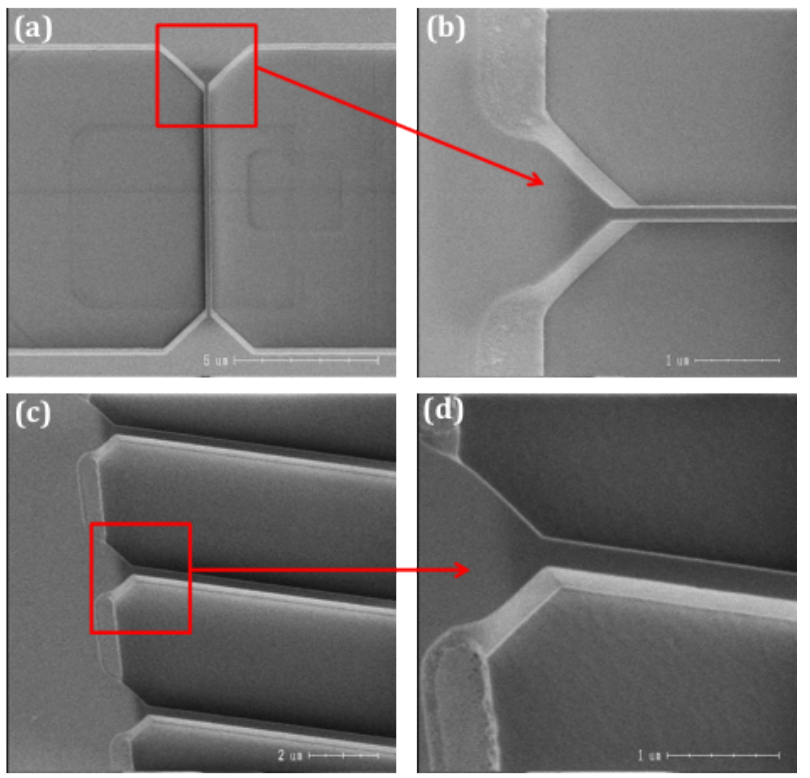

Fig. 3. SiNW-FETs device. (a) A 100nm straight sidewalls SiNW-FET with zoomed in top view ((b) shown in a red box) showing straight sidewalls. (c) Tilted SEM images of a 200nm wide SiNW FETs arrays showing with (d) zoomed in tilted view showing the smooth sidewalls.

previously performed on different samples, showed that one hour incubation was enough to have the specific coupling between antibodies, onto the NW surface, and antigens, in the electrolyte.

After each incubation, the sample was thoroughly rinsed to eliminate unreacted antigen molecules and dried under a stream of nitrogen.

Electrical characterization of the etched SiNW-FET devices was performed using a Cascade Microtech Probe station and a Hewlett-Packard 4156A precision semiconductor parameter analyzer. The electrical properties of the devices, including both current-voltage $\left(\mathrm{I}_{d s}-\mathrm{V}_{d s}\right)$ and current-back gate voltage $\left(\mathrm{I}_{d s}-\mathrm{V}_{b g}\right)$ characteristics, were measured in air before and after the incubation in VEGF.

\section{RESULTS AND DISCUSSION}

\section{A. SEM imaging}

Fig. 3 presents the SEM images of the fabricated SiNWFETs. Both high quality NWs and arrays of NWs were manufactured and used for biosensing. The described topdown fabrication process showed good control on dimensions and led to devices with multiple surfaces of $\{100\}$ crystalline planes [17]. Tilted SEM images show smooth and straight sidewalls, factors that helped in improving the quality of the functionalization process.

\section{B. Electrical measurements}

Data reported in Fig. 4 were acquired on one SiNW-FET at a fixed source to drain voltage $\mathrm{V}_{d s}$ of $2 \mathrm{~V}$. Blue, black and red curves represent the measurements performed on the same device before the surface modification, after the incubation

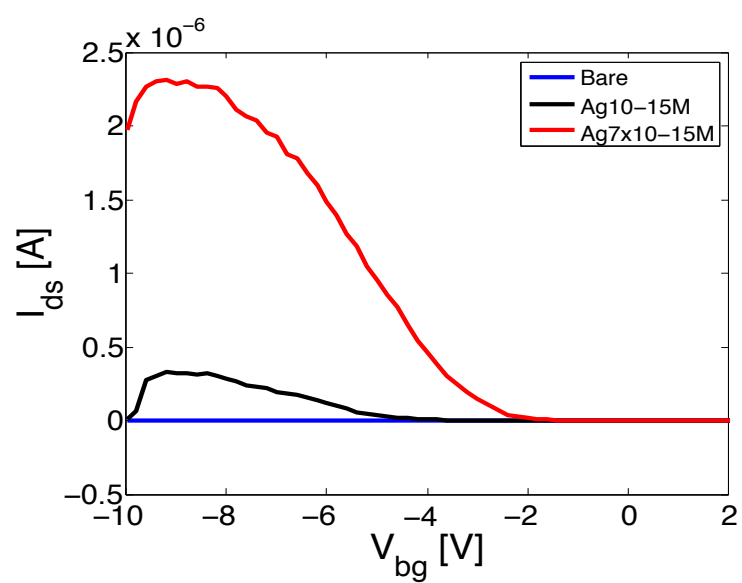

Fig. 4. $\mathrm{I}_{d s}$ vs $\mathrm{V}_{b g}$ characteristics acquired on one of the fabricated single NWs at $\mathrm{V}_{d s}=2 \mathrm{~V}$. Data were acquired for different VEGF concentrations and confirm the increasing threshold voltage.

with 1fM VEGF, and after the incubation with 7fM VEGF, respectively. The gate dependence of the biosensors clearly showed a change. The threshold voltage $\left(\mathrm{V}_{T}\right)$ of the NW device shifted towards less negative $\mathrm{V}_{b g}$ values as proof of the chemical gating effect deriving from the immuno-recognition events between anti-VEGF and VEGF molecules on P-type nanowires [15], [21]. We also consistently observed enhanced conductance in NW-FETs after antigen uptake and increasing concentrations of VEGF, as confirmed by data in Fig. 5. The conductance points reported in the graph were calculated as the slope of the $\mathrm{I}_{d s}-\mathrm{V}_{d s}$ characteristic. Error bars stand for the standard deviation calculated on repeated estimates of the current slope.

Increased conducibility and gating effect were found in a coherent and reproducible manner not only in single NWs but in arrays of NW-FETs too.

These results are in agreement with the state-of-the-art of FET based biosensors [22], and confirm the occurring detection of vascular endothelial growth factor molecules. Moreover, they show that the increased Debye length deriving from the absence of the solution counterions when measuring in air, enables the sensing of antigen molecules in a very low concentration range (fM). This range is compatible with the ultra small amounts of pathogenic factors characterizing biological events at the very early stage of the disease. Thus, the developed SiNW-FET device shows great promise for the highly sensitive and selective detection for health care. The integration of the NW based biosensors in medical devices can significantly advance the sensing performances of current early diagnosis tools.

\section{CONClusion}

In this paper, we have presented a SiNW-FET biosensors fabricated via IC/CMOS compatible process and used for real-time, specific, label-free and highly sensitive immunodetection. The FET devices incorporate high quality NWs with straight and smooth sidewalls, highly compatible with 


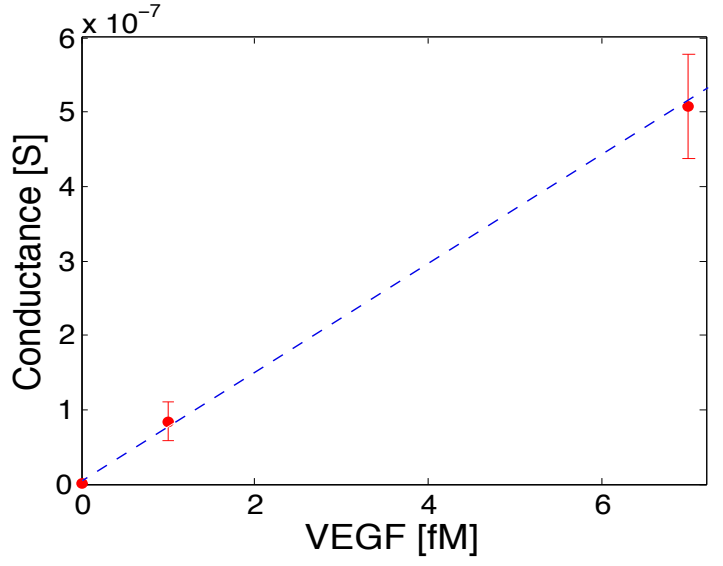

Fig. 5. Corresponding NW-FET conductance as function of the VEGF concentration. Error bars stand for the standard deviation calculated on repeated estimates of the current slope.

high performance functionalization process of surfaces with biomolecular species. The wire surface is modified with antibody probes for sensing vascular endothelial growth factor analytes. VEGF plays a critical role in the pathological angiogenesis that occurs in a number of diseases, including cancer. The electrical properties of the NWs before and after the uptake of increasing concentrations of VEGF are acquired in humid air. The results demonstrate increasing wire conductance properties as function of antigen concentrations, in good agreement with data currently reported in the literature. The innovating approach of measuring on dried samples after occurred uptake events between anti-VEGF and VEGF has shown enhanced sensing performances by getting rid of the Debye screening issue and leading to detection in the really low concentration range of femto molar.

\section{ACKNOWLEDGMENT}

This work has been supported by the multidisciplinary FNS grant (CR32I3 135073) and by an ERC grant (ERC-2009-Adg246810).

\section{REFERENCES}

[1] A. Poghossian, M. Abouzar, F. Amberger, D. Mayer, Y.Han, S. Ingebrandt, A. Offenhäusser, and M. Schöning, "Field-effect sensors with charged macromolecules: Characterisation by capacitancevoltage, constant-capacitance, impedance spectroscopy and atomic-force microscopy methods," Biosens. Bioelectron., vol. 22, pp. 2100-2107, 2007.

[2] Y. Cui, X. Duan, J. Hu, and C. M. Lieber, "Doping and electrical transport in silicon nanowires," Acc. Chem. Res., vol. 104, no. 22, pp. 5213-5216, 2000

[3] R. K. Iler, The Chemistry of Silica. New York: Wiley, 1979.

[4] P. N. Barlett, "Modification of sensor surfaces," in Handbook of Chemical and Biological Sensors, J. S. . Schultz and R. F. . Taylor, Eds. University of Pittsburgh, Pennsylvania, USA: IOP Publishing Ltd., 1996.

[5] Y. Cui, Q. Wei, H. Park, and C. M. Lieber, "Nanowire nanosensors for highly sensitive and selective detection of biological and chemical species," Science, vol. 209, no. 5533, pp. 1289-1292, 2001.

[6] C. Lieber and J. i. Hahm, "Direct ultrasensitive electrical detection of DNA and DNA sequence variations using nanowire nanosensors," Nano Lett., vol. 4, no. 1, pp. 51-54, 2004.

[7] S. Carrara, A. Cavallini, E. C. Y. Maruyama, and G. D. Micheli, "A new ethylene glycol-silane monolayer for highly-specific dna detection on silicon chips," Surf. Sci. Lett., vol. 604, pp. L71-L74, 2010.
[8] A. Hoeben, B. Landuyt, M. Highley, H. Wildiers, A. V. Oosterom, and E. D. Bruijn, "Vascular endothelial growth factor and angiogenesis," Pharmacol Rev., vol. 56, no. 4, pp. 549-580, 2004.

[9] Y. A. Muller, H. W. Christinger, B. A. Keyt, and A. M. de Vos, "The crystal structure of vascular endothelial growth factor (VEGF) refine to 1.93 å resolution: multiple copy flexibility and receptor binding," Structure, vol. 5, no. 10, pp. 1325-1338, 1997.

[10] O. Knopfmacher, A. Tarasov, W. Fu, M. Wipf, B. Niesen, M. Calame, and C. Schönberger, "Nernst limit in dual-gated Si-nanowire FET sensors," Nano Lett., vol. 10, no. 6, pp. 2268-2274, 2010.

[11] G.-J. Zhang, G. Zhang, J. H. Chua, R.-E. Chee, A. A. E. H. Wong, K. D. Buddharaju, N. Singh, Z. Gao, and N. Balasubramanian, "DNA sensing by silicon nanowire: charge layer distance dependence," Nano Lett., vol. 8, no. 4, pp. 1066-1070, 2008.

[12] E. Stern, R. Wagner, F. J. Sigworth, R. Breaker, T. M. Fahmy, , and M. A. Reed, "Importance of the Debye Screening Length on Nanowire Field Effect Transistor Sensors," Nano Lett., vol. 7, no. 11, pp. 34053409, 2007.

[13] S. Carrara, D. Sacchetto, M.-A. Doucey, C. Baj-Rossi, G. D. Micheli, and Y. Leblebici, "Memristive-biosensors: A new detection method by using nanofabricated memristors," Sens. Actuators, B, vol. 171172, no. 0, pp. $449-457,2012$.

[14] F. Puppo, A. Dave, , M.-A. Doucey, D. Sacchetto, C. Baj-Rossi, Y. Leblebici, G. D. Micheli, and S. Carrara, "Memristive biosensors under varying humidity conditions," IEEE Trans. Nanobiosci., submitted for publication.

[15] C.-C. Tsai, P.-L. Chiang, C.-J. Sun, T.-W. Lin, Ming-HsuehTsai, Y.C. Chang, and Y.-T. Chen, "Surface potential variations on a silicon nanowire transistor in biomolecular modification and detection," Nanotechnology, vol. 22, p. 135503, 2011.

[16] T. S. Y. Moh, P. G., de Smet, L.C.P.M., van Rijn, S. C.J.M, E.J.R., and P. Sarro, "Fabrication of nanowires for biosensing applications," in Selected Publications from Symposium of Nanodevices and Nanofabrication in ICMAT2011, Q. Zhang, W. I. Milne, and J. Zou, Eds. Singapore: Pan Stanford Publishing Pte. Ltd, 2012.

[17] T. S. Y. Moh, Y. Maruyama, C. Shen, G. Pandraud, L. D. Smet, H. Tong, C. Van Rijn, E. Sudholter, and P. Sarro, "IC compatible top down process for silicon nanowire FET arrays with three $\{100\}$ surfaces for (BIO) chemical sensing," in Solid-State Sensors, Actuators and Microsystems Conference (TRANSDUCERS), 2011 16th International, 2011, pp. 15901593.

[18] T. S. Y. Moh, S. K. Srivastava, S. Milosavljevic, M. Roelse, G. Pandraud, H. Zandbergen, L. C. P. M. De Smet, C. van Rijn, E. Sudholter, M. A. Jongsma, and P. Sarro, "Silicon nanowire fet arrays for real time detection of chemical activation of cells," in Micro Electro Mechanical Systems (MEMS), 2012 IEEE 25th International Conference on, 2012, pp. 1344-1347.

[19] F. D. A.J.W. Kusnezow, A. Walijew and J. Hoheisel, "Antibody microarrays: an evaluation of production parameters," Proteomics, vol. 3, no. 3, pp. 254-264, 2003.

[20] H. J. D. Kim, H.G. Lee and S. Kang, "Single-protein molecular interactions on polymer-modified glass substrates for nanoarray chip application using dual-color TIRFM,' Bull. Korean Chem. Soc., vol. 28, no. 5, pp. 783-790, 2007.

[21] C. Li, M. Curreli, H. Lin, B. Lei, F. N. Ishikawa, R. Datar, R. J. Cote, M. E. Thompson, and C. Zhou, "Complementary detection of prostatespecific antigen using $\mathrm{in}_{2} \mathrm{O}_{3}$ nanowires and carbon nanotubes," $J$. AM. Chem. Soc., vol. 127, pp. 12 484-12 485, 2005.

[22] K.-I. Chen, B.-R. Li, and Y.-T. Chen, "Silicon nanowire file-effect transistor-based biosensors for biomedical diagnosis and cellular recording investigation," Nano Today, vol. 6, pp. 131-154, 2011. 